\title{
CARACTERIZACIÓN DEL TRAUMA PEDIÁTRICO EN UN HOSPITAL MILITAR DE LA CIUDAD DE BOGOTÁ
}

\author{
LAURA WILCHES BORNACELLII ${ }^{1}$, ADRIANA CAROLINA BARBOSA ÁLVAREZ², JORGE FLÓREZ \\ ÁLVARO COGOLLOS AMAYA ${ }^{4}$, GLORIA FLÓREZ MEDINA ${ }^{5}$
}

\author{
${ }^{1} M D$ Especialista en Pediatra. Especialista en Docencia Universitaria. \\ Profesor Asistente Universidad Militar Nueva Granada. Coordinadora Servicio de Urgencias Pediátricas Hospital \\ Militar Central. Bogotá, Colombia. \\ ${ }^{2}$ Médico, Residente de Pediatría, Universidad Militar Nueva Granada. \\ Bogotá, Colombia. \\ ${ }^{3}$ Médico, Residente de Pediatría, Universidad Militar Nueva Granada. \\ Bogotá, Colombia. \\ ${ }^{4}$ Médico Cirujano, Grupo de Trauma, Hospital Militar Central. \\ Bogotá, Colombia. \\ ${ }^{5}$ Co (RA) Enf. Coordinadora Administrativa Grupo de Trauma. Bogotá, Colombia.
}

Correspondencia: laurawilchesmd@gmail.com

Recibido: Enero 13 de 2015 Aceptado: Mayo 27 de 2015

\begin{abstract}
Resumen
Objetivos: Describir las características sociodemográficas y clínicas de los pacientes entre 0 y 15 años de edad hospitalizados en el 2011 por trauma Pediátrico en el Hospital Militar Central.

Métodos: Se realizó un estudio retrospectivo descriptivo en los pacientes pediátricos del Hospital Militar Central de Bogotá, comprendido entre el 1 de enero de 2.011 y 31 de diciembre de 2.011. De un total de 1277 hospitalizados por trauma se tomaron 92 pacientes con edades comprendidas entre los 0 y 15 años de edad, de los cuales precisaron ser ingresados 81 y se incluyeron en la base de datos; con el fin de describir las características epidemiológicas de este grupo de pacientes, determinar la severidad del trauma, y si estuvo relacionado con mortalidad; así como la época del año y el horario con más prevalencia de trauma de la población estudio.

Resultados: Se encontró un predominio de trauma en los varones en una relación 1.45:1 siendo el grupo de edad comprendido entre los 11- 15 años el más frecuente, con un total de 21 pacientes (26\%). El 71.6\% (58 casos) de los ingresos tuvieron un índice de trauma pediátrico de 8 o superior. No encontramos mortalidad asociada al trauma en la casuística estudiada.

Conclusiones: El estudio permitió conocer los aspectos sociodemográficos y clínicos de la población pediátrica que se trata en el Hospital Militar Central, y los puntos a intervenir en la institución con el fin de prestar una atención de calidad en la asistencia hospitalaria; así como los posibles factores pronósticos precoces para disminuir la prevalencia de dicha patología.
\end{abstract}

Palabras clave: Trauma Pediátrico, Índice de trauma pediátrico, características epidemiológicas de trauma pediátrico. 


\title{
CHARACTERIZATION OF PEDIATRIC TRAUMA IN A MILITARY HOSPITAL OF BOGOTÁ
}

\begin{abstract}
Objectives: To describe the sociodemographic and clinical characteristics of patients between 0 and 15 years of age hospitalized on 2011 due to a Pediatric trauma within the Hospital Militar Central.

Methods: A retrospective descriptive study was performed on pediatric patients of the Hospital Militar Central of Bogota, between January 1, 2011 and December 31, 2011. From a total number of 1277 hospitalized patients due to trauma, 92 patients were taken with ages ranging from 0 to 15 years of age, from which 81 were indicated as admitted and they were included within the database; in order to describe the epidemiological characteristics of this group of patients, by determining the severity of the trauma, and if it was related to mortality; as well as the time of the year and the Schedule with the most prevalence of the trauma of the study population.

Results: A predominance was found of trauma on male with a rate of $1.45: 1$ with the most frequent age group ranging from 11 to 15 years of age, with a total of 21 patients (26\%). $71.6 \%$ (58 cases) from the admitted patients had a pediatric trauma index of 8 or higher. We did not find mortality associated to trauma within the studied casuistry.

Conclusions: The study allowed to recognize the sociodemographic and clinical aspects of the pediatric population that is treated within the Hospital Militar Central, and the points to be intervened within the institution in order to provide a high quality care within hospital assistance; as well as the potential early prognosis to reduce the prevalence of the pathology mentioned before.

Keywords: Pediatric trauma, Pediatric Trauma Index, epidemiological characteristics of pediatric trauma.
\end{abstract}

\section{CARACTERIZAÇÃO DE TRAUMA PEDIÁTRICO EM UM HOSPITAL MILITAR NA CIDADE DE BOGOTÁ}

\begin{abstract}
Resumo
Objetivos: Descrever as características sociodemográficas e clinicas dos pacientes entre 0 e 15 anos de idade internados no 2011 por causa do trauma pediátrico no Hospital Militar Central.

Métodos: Um estudo retrospectivo e descritivo foi realizado nos pacientes pediátricos do Hospital Central de Bogotá, a partir de 01 de janeiro de 2011 até 31 de Dezembro de 2011. De um total de 1277 internados por trauma, 92 pacientes com idades compreendidas entre 0 e 15 anos de idade foram usados para o estudo, dos quais foi preciso incluir 81 deles na base de dados, com a finalidade de descrever as características epidemiológicas deste grupo de pacientes, determinar a gravidade do trauma e se teve alguma relação com o trauma, além da época do ano com maior prevalência de trauma na população de estudo.

Resultados: Uma prevalência de trauma foi encontrada em homens numa proporção 1.45:1, com maior frequência num grupo com idades compreendidas entre os 11 e 15 anos, com um total de 21 pacientes (26\%). O 71.6\% (58) dos ingressos tiveram um índice de trauma pediátrico de 8 ou superior. Não foi encontrada nenhuma mortalidade associada com trauma dos casos estudados.

Conclusões: O estudo permitiu conhecer os aspectos sociodemográficos e clínicos da população pediátrica que está no Hospital Militar Central, bem como os pontos para intervir na instituição com o fim de prover cuidados de qualidade, além dos possíveis fatores prognósticos iniciais para diminuir a prevalência desta patologia.
\end{abstract}

Palavras-chave: Trauma pediátrico, índice do trauma pediátrico, as características epidemiológicas do trauma pediátrico. 


\section{Introducción}

El trauma pediátrico es una de las patologías que tiene mayor impacto social y económico (1); siendo de vital importancia no sólo conocer a profundidad las medidas de soporte y manejo del mismo, sino la estrategia de prevención a todo nivel para así disminuir su incidencia.

Colombia es uno de los países con más altos índices de violencia a nivel mundial, en el que a diario se reportan miles de víctimas de diferentes edades por conflicto armado, colocando el trauma en nuestro país como una de las cinco primeras causas de muerte en sujetos menores de 40 años. $(2,3)$.

Según estadísticas del DANE y del Instituto Nacional de Medicina Legal, la tasa de mortalidad infantil ha disminuido desde 27,48 por mil nacidos vivos en 1998 hasta 20,1 por mil nacidos vivos en el 2009; sin embargo dentro de las cinco primeras causas de mortalidad en hombres de 1 a 4 años en el 2009, aparecen el ahogamiento y los accidentes de transporte como tercera y cuarta causa de muerte respectivamente, después de las infecciones respiratorias y las malformaciones congénitas. Durante el mismo año en la población de 5 a 14 años, los accidentes de transporte terrestre fueron la primera causa de mortalidad en hombres y la segunda en mujeres; y en el grupo de 15 a 44 años, las agresiones (homicidios) inclusive su secuelas, es la principal causa de defunción en hombres, seguido de los accidentes en transporte terrestre (4).

En el 2013 las personas más afectadas por los accidentes de transporte son los hombres con edad comprendida entre los 15 y 34 años, representando el $43.7 \%$ del total de las muertes (5).

Tabla 1. Muertes por accidentes de transporte según grupo de edad y sexo de la víctima. Colombia 2013. Tomado de (5).

\begin{tabular}{|c|c|c|c|c|c|c|c|c|c|c|c|c|}
\hline \multirow{2}{*}{$\begin{array}{c}\text { GRUPO } \\
\text { DE } \\
\text { EDAD }\end{array}$} & \multicolumn{4}{|c|}{ HOMBRE } & \multicolumn{4}{|c|}{ MUJER } & \multicolumn{4}{|c|}{ TOTAL } \\
\hline & CASOS & $\%$ & $\begin{array}{c}\% \\
\text { ACUMULADO }\end{array}$ & $\begin{array}{c}\text { TASA X } 100.000 \\
\text { HABITANTES }\end{array}$ & CASOS & $\%$ & $\begin{array}{c}\% \\
\text { ACUMULADO }\end{array}$ & $\begin{array}{c}\text { TASA X } 100.000 \\
\text { HABITANTES }\end{array}$ & CASOS & $\%$ & $\begin{array}{c}\% \\
\text { ACUMULADO }\end{array}$ & $\begin{array}{c}\text { TASA X } 100.000 \\
\text { HABITANTES }\end{array}$ \\
\hline$(00$ a 04) & 36 & 0,71 & 0,71 & 1,64 & 42 & 3,56 & 3,56 & 2,00 & 78 & 1,25 & 1,25 & 1,81 \\
\hline (05 a 09) & 54 & 1,07 & 1,79 & 2,48 & 34 & 2,88 & 6,44 & 1,63 & 88 & 1,42 & 2,67 & 2,06 \\
\hline (10 a 14) & 81 & 1,61 & 3,39 & 3,67 & 38 & 3,22 & 9,65 & 1,79 & 119 & 1,91 & 4,58 & 2,75 \\
\hline (15 a 17) & 193 & 3,83 & 7,23 & 14,31 & 55 & 4,66 & 14,31 & 4,26 & 248 & 3,99 & 8,57 & 9,40 \\
\hline (18 a 19) & 207 & 4,11 & 11,33 & 23,10 & 54 & 4,57 & 18,88 & 6,32 & 261 & 4,20 & 12,77 & 14,91 \\
\hline (20 a 24) & 732 & 14,53 & 25,86 & 33,88 & 110 & 9,31 & 28,20 & 5,34 & 842 & 13,54 & 26,31 & 19,94 \\
\hline (30 a 34) & 549 & 10,90 & 49,44 & 32,85 & 75 & 6,35 & 43,27 & 4,28 & 624 & 10,03 & 48,27 & 18,22 \\
\hline (35 a 39) & 379 & 7,52 & 56,97 & 25,44 & 84 & 7,11 & 50,38 & 5,31 & 463 & 7,44 & 55,72 & 15,07 \\
\hline (40 a 44) & 359 & 7,13 & 64,09 & 25,98 & 67 & 5,67 & 56,05 & 4,47 & 426 & 6,85 & 62,57 & 14,79 \\
\hline (45 a 49) & 314 & 6,23 & 70,33 & 22,98 & 69 & 5,84 & 61,90 & 4,61 & 383 & 6,16 & 68,72 & 13,38 \\
\hline (50 a 54) & 286 & 5,68 & 76,00 & 23,63 & 60 & 5,08 & 66,98 & 4,50 & 346 & 5,56 & 74,29 & 13,61 \\
\hline (55 a 59) & 249 & 4,94 & 80,94 & 25,65 & 73 & 6,18 & 73,16 & 6,76 & 322 & 5,18 & 79,47 & 15,70 \\
\hline$(60$ a 64) & 203 & 4,03 & 84,97 & 26,93 & 68 & 5,76 & 78,92 & 8,09 & 271 & 4,36 & 83,82 & 16,99 \\
\hline (65 a 69) & 201 & 3,99 & 88,96 & 35,97 & 60 & 5,08 & 84,00 & 9,44 & 261 & 4,20 & 88,02 & 21,85 \\
\hline (75 a 79) & 171 & 3,39 & 95,79 & 59,42 & 53 & 4,49 & 93,90 & 14,22 & 224 & 3,60 & 95,43 & 33,91 \\
\hline (80 y más) & 212 & 4,21 & 100,00 & 78,30 & 70 & 5,93 & 99,83 & 18,45 & 282 & 4,53 & 99,97 & 43,38 \\
\hline Total $^{*}$ & 5.038 & 100,00 & - & 21,66 & 181 & 100,00 & - & 4,95 & 6.219 & 100,00 & - & 13,20 \\
\hline
\end{tabular}


Por ello que se hace necesario redundar en esfuerzos no sólo para generar mecanismos gubernamentales con el propósito de poner fin a este conflicto; sino también para planear políticas públicas encaminadas a la prevención y el manejo integral del trauma; así como buscar optimizar los recursos humanos y estructurales de la institución.

Resulta importante describir por tal motivo, los posibles factores de riesgo que existen en el entorno de los niños, para poder estipular políticas dirigidas a todos los "cuidadores" de los menores e intervenir a tiempo en ellos. Estos factores de riesgo van desde características del medio ambiente donde se desenvuelven estos pacientes, así como de la sociedad y las actividades de las que hacen parte (2).

No existen estadísticas en nuestra institución, y hay muy pocas en el país relacionadas con las características del trauma pediátrico. El primer paso necesario para alcanzar estos objetivos consiste entonces, en que el médico pediatra conozca la caracterización del trauma pediátrico descifrando sus aspectos epidemiológicos, sociodemográficos y clínicos; a partir de una institución asistencial como la nuestra que por su nivel de complejidad, se ha convertido en un centro nacional de referencia de trauma para toda la población civil donde se desconoce hasta el momento la incidencia real, la prevalencia y las características de la población que lo padece. Éstas fortalezas permitirán facilitar la recolección y análisis de una casuística propia de las Fuerzas Militares, que servirá de punto de referencia a nivel Nacional para posteriormente consolidarnos como país, estableciendo unas buenas prácticas médicas y de vigilancia que puedan llegar incluso a ser un referente Internacional.

Este estudio pretende describir las características sociodemográficas y clínicas de los pacientes entre 0 y 15 años de edad hospitalizados durante el 2011 por trauma Pediátrico en el Hospital Militar Central; año en el cual se recopiló una base de datos completa por el grupo de Trauma de nuestra Institución. Además, pretende caracterizar los mecanismos del trauma, los sitios anatómicos más frecuentemente afectados, temporalidad y frecuencia; de la misma manera determinar la mortalidad y los días de hospitalización que a causa del trauma pediátrico se sucedieron en los pacientes blanco de este estudio. Finalmente establecer las condiciones prehospitalarias que se prestaron a estos pacientes antes de su llegada al Hospital Militar Central durante el 2011.

\section{Materiales y métodos}

Se realizó un estudio descriptivo retrospectivo en el servicio de Pediatría del Hospital

Militar Central de Bogotá. La población estudiada fueron los pacientes hospitalizados por trauma pediátrico entre 0 y 15 años en esta institución en un periodo de 1 año, desde el 1 de enero del 2011 hasta el 31 de diciembre del 2011. Se definió Trauma pediátrico como una lesión o herida estructural $y / o$ fisiológica, secundaria a una exposición aguda a energía mecánica, térmica, química o eléctrica en pacientes pediátricos.

A partir de una revisión de la base de datos del Grupo de Trauma del Hospital Militar Central de Bogotá año 2011 que incluyó 1277 pacientes con diagnóstico de trauma, se tomaron los pacientes con edades comprendidas entre los 0 y 15 años hospitalizados con diagnóstico de trauma pediátrico. Se registraron en la base de datos 98 pacientes de los cuales se excluyeron 17 por presentar historias clínicas incompletas o con diagnóstico no pertinente. Se analizaron 81 casos de trauma pediátrico.

\section{Criterios de inclusión}

- Historias Clínicas de pacientes entre 0-15 años hospitalizados por trauma pediátrico durante el año 2011.

\section{Criterios de Exclusión}

- Historias clínicas de pacientes entre 0- 15 años hospitalizados por trauma pediátrico durante el año 2011 en quienes no fue posible acceder a su historia clínica.

- Historias clínicas de pacientes entre 0-15 años cuyas historias clínicas o registros estén incompletos.

Una vez obtenida esta salida se procedió, con las historias clínicas seleccionadas, a la captura de datos en forma electrónica o no de las variables establecidas, y se exportó a una tabla de Excel como instrumento de tabulación. Esta información fue migrada a un paquete estadístico Stata Versión 12.0 para su respectivo análisis. Las variables continuas se presentaron como medias, desviación estándar o medianas con rango intercuartílico según sea apropiado. Las variables categóricas se presentaron como porcentajes.

Las variables cuantitativas se presentaron en histogramas de frecuencias o en tablas de frecuencias. Las variables cualitativas se presentaron en gráficas de barras o sectores, o en tablas de frecuencias absolutas - relativas. 
Resultados

Tabla 2. Características sociodemográficas de los pacientes con trauma pediátrico del HOMIC 2011.

\begin{tabular}{|c|c|c|}
\hline & Sujetos & \\
\hline & $\mathbf{N}$ & $\%$ \\
\hline \multicolumn{3}{|l|}{ Sexo } \\
\hline Masculino & 48 & 59,3 \\
\hline Femenino & 33 & 40,7 \\
\hline \multicolumn{3}{|l|}{ Grupo Etario } \\
\hline Lactante Menor & 5 & 6,2 \\
\hline Lactante Mayor & 17 & 21,0 \\
\hline Preescolares & 20 & 24,7 \\
\hline Escolares & 18 & 22,2 \\
\hline Adolescentes & 21 & 25,9 \\
\hline \multicolumn{3}{|l|}{ Fuerza militar } \\
\hline Ejército & 65 & 80,2 \\
\hline Fuerza Aérea & 7 & 8,6 \\
\hline Armada & 5 & 6,2 \\
\hline \multicolumn{3}{|l|}{ Grado militar } \\
\hline Hijos de suboficiales & 41 & 50,6 \\
\hline Hijos de soldados & 30 & 37,0 \\
\hline Hijos de oficiales & 8 & 9,9 \\
\hline Hijos de civiles & 2 & 2,5 \\
\hline \multicolumn{3}{|l|}{ Zona de procedencia } \\
\hline Urbano & 66 & 81,5 \\
\hline Rural & 15 & 18,5 \\
\hline \multicolumn{3}{|c|}{ Departamento de procedencia } \\
\hline Cundinamarca & 51 & 63,0 \\
\hline Bogotá & 7 & 8,6 \\
\hline Boyacá & 5 & 6,2 \\
\hline Meta & 4 & 4,9 \\
\hline Tolima & 4 & 4,9 \\
\hline Huila & 3 & 3,7 \\
\hline Caquetá & 2 & 2,5 \\
\hline Casanare & 1 & 1,2 \\
\hline Putumayo & 1 & 1,2 \\
\hline Norte de Santander & 1 & 1,2 \\
\hline Guaviare & 1 & 1,2 \\
\hline \multicolumn{3}{|l|}{ Lugar de ocurrencia } \\
\hline Extradomiciliario & 48 & 59,3 \\
\hline Domiciliario & 33 & 40,7 \\
\hline
\end{tabular}

En esta tabla se describe la población incluida en el presente trabajo.

La mayor proporción de casos de trauma pediátrico fue en adolescentes de sexo masculino; pertenecientes en su mayoría al Ejército, con procedencia urbana del departamento de Cundinamarca.

Más de la mitad de los casos ocurrieron fuera de casa en su mayoría en sitios públicos. Con respecto a los intradomiciliarios, predominaron los traumas accidentales.

Ocurrieron 9 accidentes de tránsito (11.1\%) de los cuales tres fueron como peatón, dos como ocupante de moto, dos ocupantes de vehículo, un caso como ocupante en trasporte público y otro caso como motociclista.

Tabla 3. Características clínicas de los pacientes con trauma pediátrico del HOMIC 2011.

\begin{tabular}{|c|c|c|}
\hline & Sujetos & \\
\hline & $\mathbf{N}$ & $\%$ \\
\hline \multicolumn{3}{|l|}{ Sitio anatómico del trauma } \\
\hline Extremidades & 45 & 55,6 \\
\hline Craneoencefalico & 16 & 19.8 \\
\hline Tórax & 6 & 7,4 \\
\hline Cara & 4 & 4,9 \\
\hline Abdomen & 2 & 2,5 \\
\hline Politraumatismo & 2 & 2,5 \\
\hline \multicolumn{3}{|l|}{ Tejido comprometido } \\
\hline Tejido Óseo / Fracturas & 31 & 38,3 \\
\hline Tejidos blandos & 29 & 35,8 \\
\hline Tejidos blandos y visceral & 6 & 7,4 \\
\hline Tejidos blandos y óseo & 4 & 4,9 \\
\hline Tejido Óseo y visceral & 3 & 3,7 \\
\hline $\begin{array}{l}\text { Tejido blando, visceral y } \\
\text { fracturas }\end{array}$ & 2 & 2,5 \\
\hline \multicolumn{3}{|l|}{ Glasgow } \\
\hline $15 \backslash 15$ & 71 & 87,7 \\
\hline $14 \backslash 15$ & 3 & 3,7 \\
\hline $12 \backslash 15$ & 2 & 2,5 \\
\hline 8115 & 1 & 1,2 \\
\hline $7 \backslash 15$ & 1 & 1,2 \\
\hline Bajo sedación & 1 & 1,2 \\
\hline No dato & 2 & 2,5 \\
\hline \multicolumn{3}{|l|}{ Vía aérea } \\
\hline Permeable & 74 & 91,3 \\
\hline No Permeable & 5 & 6,2 \\
\hline No Dato & 2 & 2,5 \\
\hline
\end{tabular}


Dentro de las características clínicas, las fracturas simples de las extremidades fueron las lesiones más frecuentes, seguido por el trauma craneoencefálico.

En su gran mayoría ingresaron al servicio de Urgencias pediátricas en transporte terrestre particular, hemodinámicamente estable, con Glasgow 15/15 y vía aérea permeable. Los que requirieron hospitalización tuvieron una corta estancia hospitalaria, en su mayoría menos de 7 días (61,7\%).

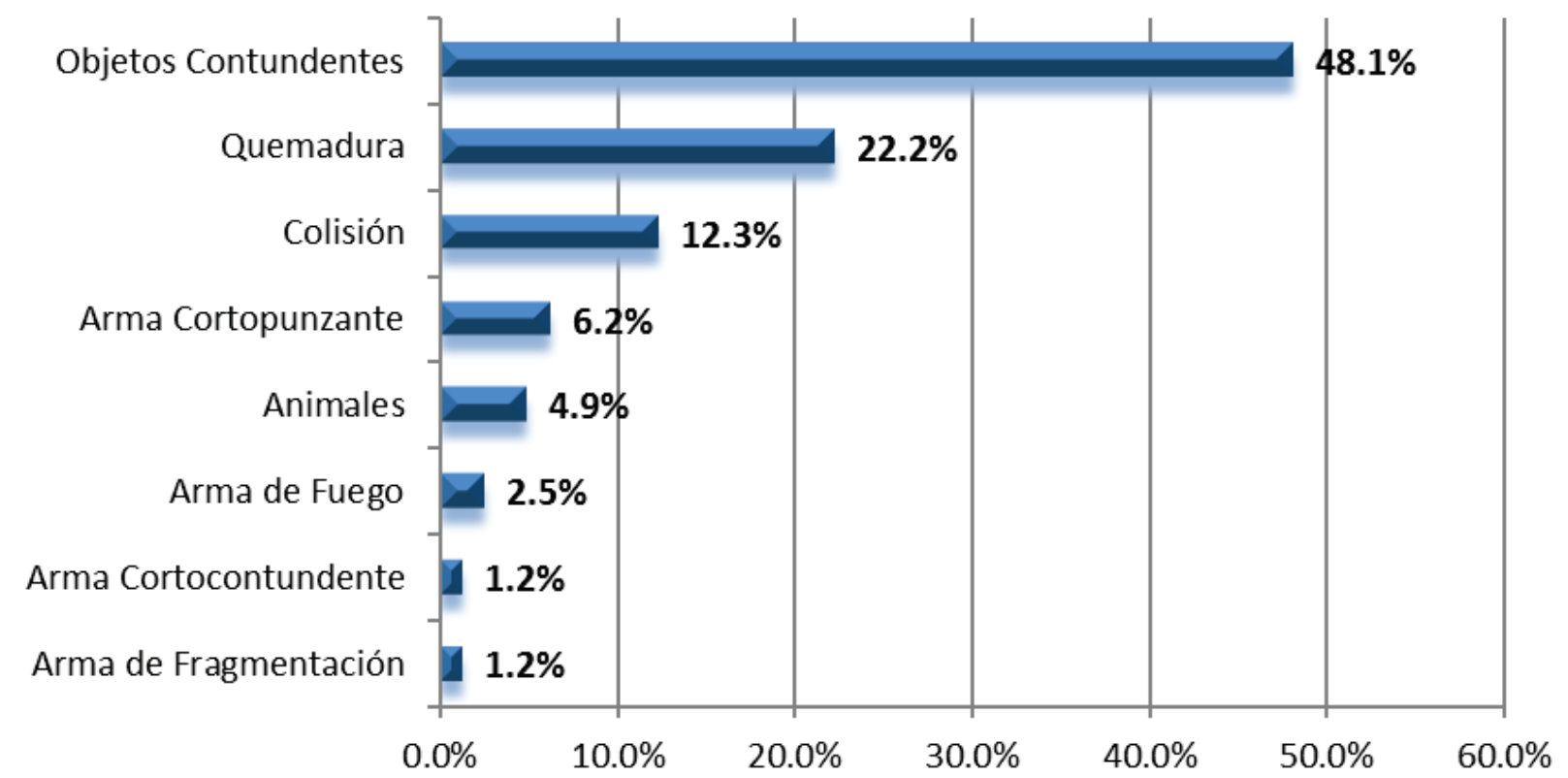

Figura 1. Distribución de acuerdo a mecanismo del trauma.

El principal mecanismo de trauma fue el contundente, seguido por las quemaduras. La mayoría de las quemaduras fueron múltiples, seguidas por compromiso de miembros superiores y cabeza.

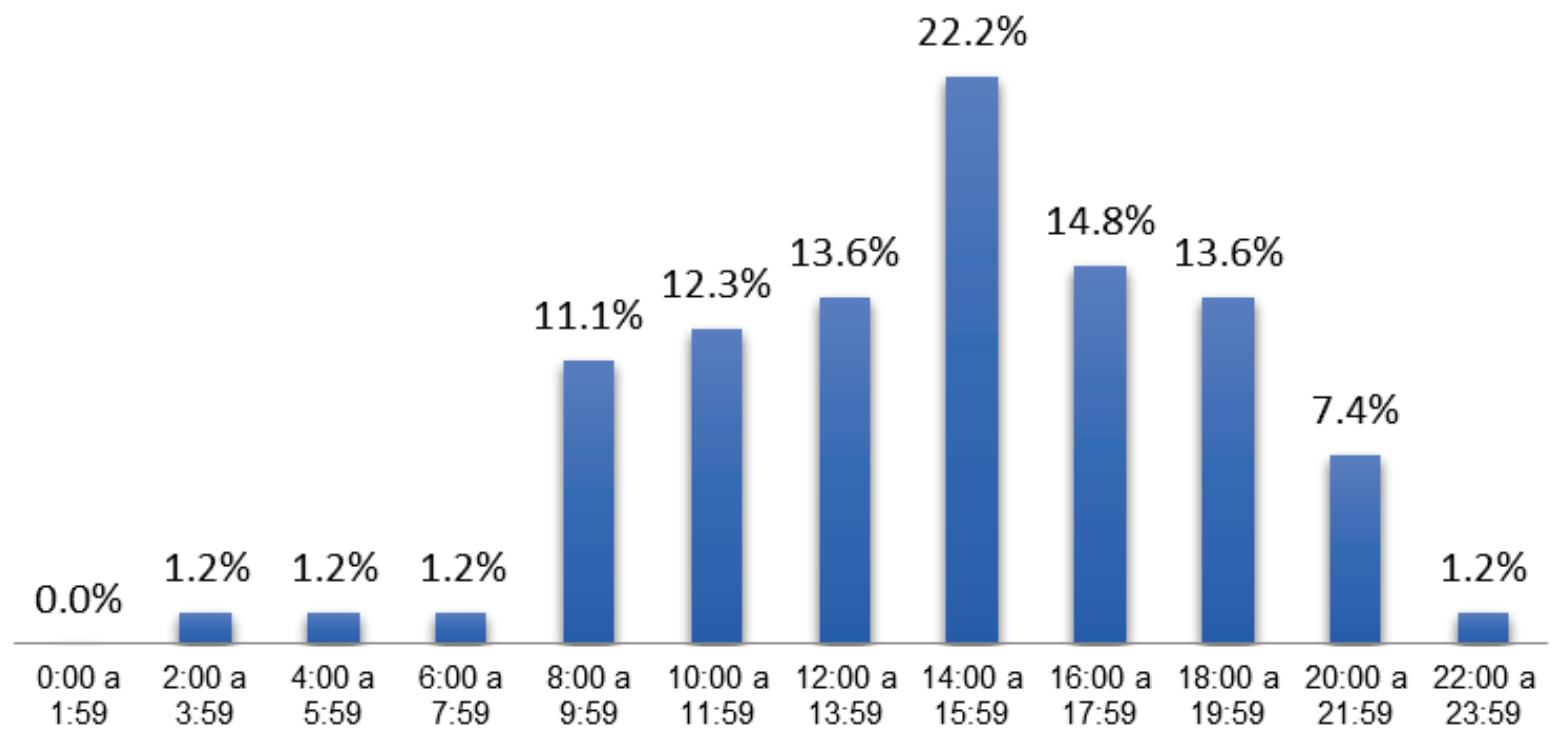

Figura 2. Distribución de la presentación de los traumas de acuerdo a la hora del día. 


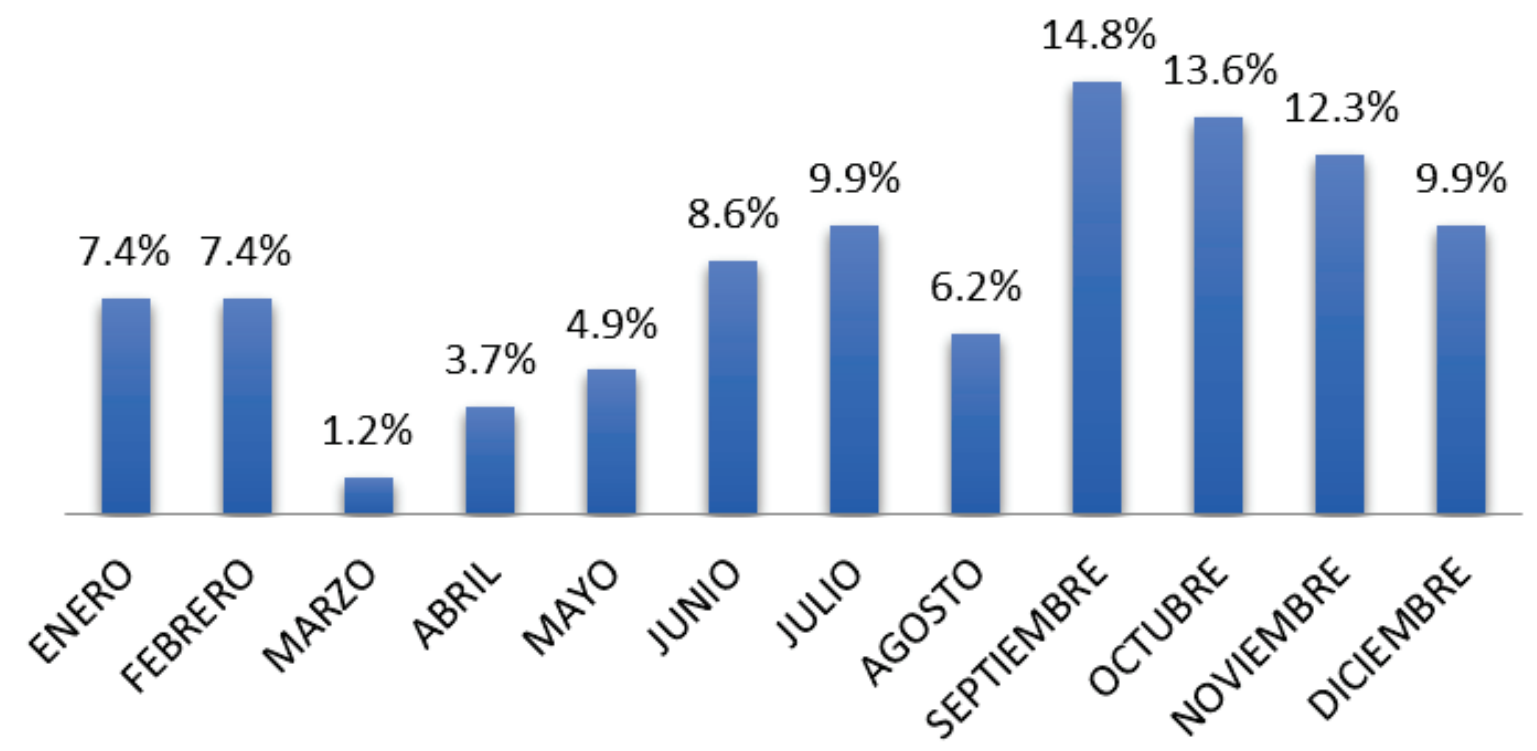

Figura 3. Distribución de la presentación de los traumas por mes.

El momento del día con mayor frecuencia de trauma fue en los últimos cuatro meses del año, especialmente en horas de la tarde.

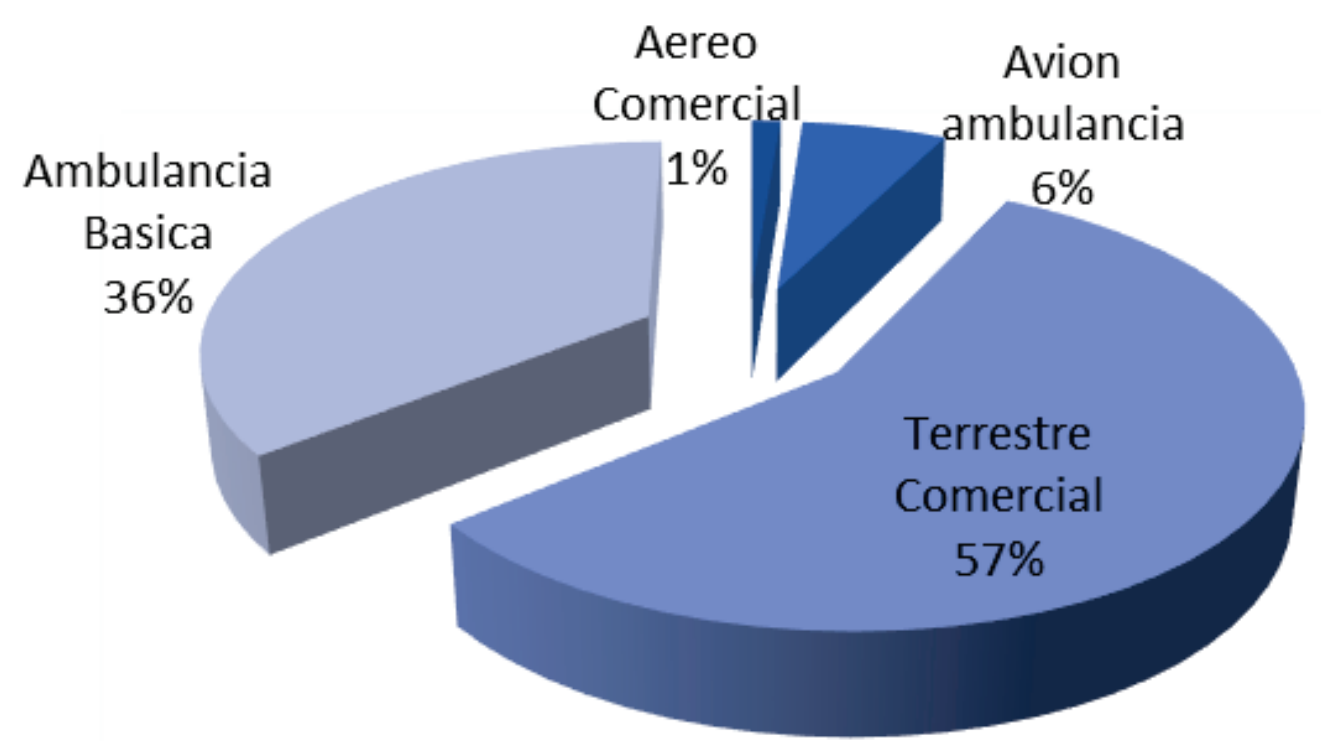

Figura 4. Distribución de acuerdo al tipo de trasporte pre hospitalario. 
Tabla 4. Índice de trauma pediátrico para mortalidad y pronóstico. Modificado de Navascués del Ríos J.A, et al. Estudio Epidemiológico de los accidentes en la infancia: Primer Registro de Trauma Pediátrico. An Pediatric. 1997;47(4).

\begin{tabular}{|l|l|l|l|}
\hline PUNTUACIÓN & \multicolumn{1}{|c|}{+2} & \multicolumn{1}{c|}{+1} & \multicolumn{1}{c|}{-1} \\
\hline PESO & $>20 \mathrm{Kg}$ & $10-20 \mathrm{~kg}$ & $<10 \mathrm{Kg}$ \\
\hline VÍA AÉREA & Normal & Sostenible & Insostenible \\
\hline T.A & $>90 \mathrm{~mm} \mathrm{Hg}$ & $90-50 \mathrm{~mm} \mathrm{Hg}$ & $<50 \mathrm{~mm} \mathrm{Hg}$ \\
\hline S.N.C & Consciente & Obnubilado & Coma o descerebrado \\
\hline HERIDAS & No & Menores & Mayor o penetrante \\
\hline FRACTURAS & No & Cerrada & Abierta o múltiple \\
\hline
\end{tabular}

La probabilidad de muerte comienza a partir de un ITP de 8 y aumenta progresivamente cuanto menor es la puntuación; de tal forma que un ITP negativo se acerca al $100 \%$ de mortalidad $(2,8)$. El ITP también es útil como criterio de selección de pacientes según su pronóstico y como escala a emplear para la formación de grupos epidemiológicos comparables $(8,9)$.

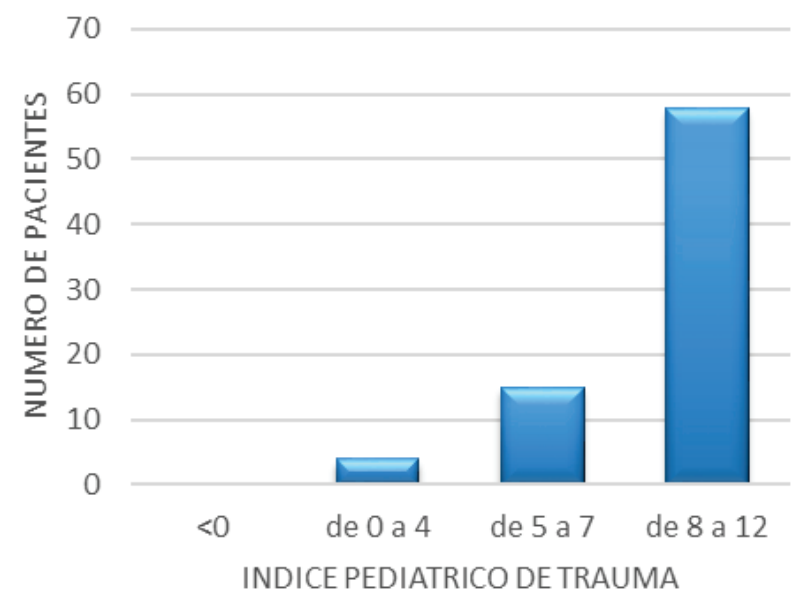

En 58 casos $(71,6 \%)$ se encontró un puntaje igual o superior a 8, en 19 casos (23.4\%) un puntaje menor a 8. Sólo se produjo un fallecimiento no atribuido al trauma durante el período estudiado.

\section{Discusión}

La literatura mundial menciona que el trauma sigue siendo la tercera causa global de muerte y la primera en Colombia en los menores de 40 años (3), representando entre el 40 al $50 \%$ de las mismas (10,5); siendo el $13 \%$ de los ingresos en un hospital pediátrico y el 25\% de los ingresos en una UCI pediátrica. Las secuelas afectan al 32\% de los niños que sufren un traumatismo grave y sobreviven, y sólo en EE.UU. esto supone unos 100.000 niños al año (11).
La repercusión social de la incapacidad infantil posttraumática es de gran magnitud, hasta el punto de que el gasto económico en recursos y personal producido por los mismos es mucho mayor que el derivado del trauma en el adulto (10).

La incidencia y la gravedad de las lesiones por trauma en la infancia requieren por ello el desarrollo de programas especiales para su prevención, atención y manejo; precisando así un eficaz sistema de asistencia en el lugar del accidente y de medios de evacuación rápidos por personal experto; así como de centros con la infraestructura y la experiencia para el manejo del niño traumatizado (12).

Para garantizar la formación del personal involucrado en el trauma se diseñaron en EE.UU. a finales de la pasada década, los cursos de entrenamiento "Advanced Trauma Life Support” (ATLS) del American College of Surgeons (13), que han sido impartidos a más de 130.000 médicos de todo el mundo. Siguiendo este modelo y con el fin de adecuar el contenido de dichos cursos a la práctica pediátrica, se pusieron en marcha en Argentina los cursos de "Atención Inicial en Trauma Pediátrico" (10).

Adicionalmente, se hace importante recopilar datos y evaluar información de centros hospitalarios pediátricos respecto a la atención de pacientes traumatizados, para abarcar desde la posible adecuación de recursos, hasta la corrección de eventuales deficiencias en el manejo del trauma; pasando por la indicación de determinadas medidas de prevención, revisión y evaluación de tratamientos efectuados, así como estudios comparativos entre distintos centros (2).

Además podrán estos datos ayudar a enriquecer las ya existentes bases de datos multicéntricas como el National Pediatric Trauma Registry (NPTR) en EE.UU, que recopila estadísticas desde 1995, y hoy cuenta con más de 55 centros de trauma, e información de más de 35.000 pacientes (2).

En el caso de la casuística estudiada aquí, se encontró que más de la mitad de los pacientes 58 casos (71.6\%), obtuvieron un ITP mayor a 8 y sólo 19 casos (23.4\%) alcanzaron un puntaje menor a 8 siendo todos mayores a 0 ; lo que les confería un mejor pronóstico al momento de su ingreso posiblemente por el mecanismo de trauma; situación reflejada en la adecuada evolución de los pacientes al egreso, puesto que en la revisión descrita no se encontraron casos de mortalidad confirmada por trauma en este grupo de pacientes, y el único caso que falleció no se pudo establecer si su causa se asoció o no al traumatismo ya que lo hizo posterior a su egreso hospitalario. 
La necesaria existencia de centros con la infraestructura y el personal adecuados para el manejo del trauma hace que en 1990 se designen oficialmente los primeros Centros de Trauma en EE.UU. El análisis de los contenidos de la base de datos del NPTR ha demostrado con posterioridad que la supervivencia de determinados grupos de pacientes traumatizados puede llegar a ser hasta 10 veces superior cuando son referidos a este tipo de centros (2).

Este estudio fue realizado en un hospital de IV nivel que cuenta con experiencia en el manejo del trauma en el adulto, por lo que creímos necesario conocer con la mayor exactitud posible todo aquello que rodeaba al niño traumatizado que llegaba a nuestro Hospital, como parte de un proyecto del grupo de trauma de nuestra institución, destinado a mejorar la atención de estos pacientes; pues la experiencia acumulada en otros países así lo aconsejaba (11).

Resultados de esta descripción clínica y sociodemográfica son similares a los obtenidos por el estudio español de Navascués y Cols (2); como predominio del trauma en el sexo masculino en proporción 1.45:1, con mayor frecuencia de presentación en escolares y adolescentes, que podría obedecer a mayores conductas de riesgo en estos pacientes.

Comparado con resultados de estudios españoles y nacionales, se encontraron datos coincidentes entre ellos: mayor prevalencia a nivel extra domiciliario (59.3\%), con mecanismo del trauma también en su mayoría por objetos contundentes, quemaduras y precipitaciones de todo tipo. Prevalencia de los mismos sitios anatómicos con predominio del trauma en las extremidades 45 casos (55.6\%), seguido del trauma craneoencefálico 16 casos (19.8\%). El principal tejido comprometido fue el óseo 31 $\operatorname{casos}(38,3 \%)(2,3)$.

Datos como los accidentes de tránsito, ( 9 casos en nuestro estudio equivalente al 11.1\%), no resultaron ser tan frecuente como en la literatura, que lo presentan como la segunda causa de trauma extra domiciliario $(10,11)$.

En cuanto a la escala de Glasgow al momento del ingreso se encontró una mayor proporción de pacientes con puntajes de 15/15 en 71 casos (87.7\%), y en 74 casos (91.3\%) la vía aérea se encontró permeable, que puede también explicar en parte la evolución adecuada de los pacientes de este estudio y la baja severidad de los traumas.

Con respecto a la atención pre hospitalaria el transporte terrestre comercial fue el más usado por los pacientes 46 casos (57\%) y en segundo lugar la ambulancia básica 29 casos (36\%) al igual que a nivel mundial, lo cual podría obedecer a que las horas en que fueron más prevalentes las lesiones, el cuidador del menor puede llamar un traslado primario o bien desplazarse con el paciente a las instituciones de salud (14).

Otros datos, por el contrario, han resultado contradictorios, como es el caso de la mortalidad, requerimiento de hospitalizaciones prolongadas o la necesidad de cuidado crítico $(10,11)$; puesto que en este estudio la mayoría de los pacientes sólo requirieron atención hospitalaria en pisos de pediatría, con estancia hospitalaria promedio de 7.54 días. Es probable que pacientes de mayor severidad fueran atendidos en primera instancia en hospitales diferentes a nuestra institución, pudiéndose derivar de allí una atenuación en la severidad encontrada.

Los resultados del presente trabajo confirman que la época del año en donde es más frecuente el trauma son los meses de Septiembre con 12 casos (14.8\%), seguido de Octubre con 11 casos (13.6\%) y Noviembre 10 casos (12.3\%); ocurriendo 41 casos (el 50.6\%) en los últimos cuatro meses de año que contrasta con la literatura europea donde se presenta más en los meses de primavera $(1,2)$, posiblemente por tener unas condiciones climáticas más favorables para realizar actividades fuera de casa.

Se pueden aportar datos novedosos en el subsistema de las fuerzas militares al mencionar que de los múltiples usuarios de las mismas, el Ejército aportó el $80.2 \%$ de los casos seguido de la Fuerza Aérea con el 8.6\%. La principal zona de procedencia de los casos fue urbana con el $81.5 \%$ y el departamento que más contribuyó con casos fue Cundinamarca con el $63.0 \%$, posiblemente por la gran cantidad de usuarios pertenecientes al Ejército y por la cercanía del Departamento de Cundinamarca a la ciudad de Bogotá, teniendo el resto de departamentos sitios más cercanos de remisión.

Datos relevantes de nuestra casuística que pueden cooperar con las estadísticas de Estados Unidos y España es el horario vespertino en el cual se presentaron la mayor parte de los traumas, especialmente entre las 14:00 y las 15:59 (22.2\%). Por otra parte, el tiempo promedio entre el trauma y la atención hospitalaria fue de 15.08 horas (SD 31.39); datos que podría ayudar a generar medidas preventivas ya sea en los hogares o en los sitios donde cuidan a los menores; como en la atención oportuna pre y hospitalaria; encaminada a dirigir esfuerzos en prestar más recurso humano y físico en estos horarios.

Como conclusión, esta primera casuística de pacientes en el Hospital Militar Central originada de una base de datos del grupo de trauma de la institución, permitió el análisis de variables sociodemográficas relevantes a las necesidades de 
las Fuerzas Militares, y comportamiento del trauma para un régimen de excepción como lo es el nuestro; sin embargo no fue posible realizar una comparación con otros centros de atención de las Fuerzas Militares por no contar con información suficiente.

Este análisis pueden no necesariamente representar las características del trauma en niños que no son usuarios de las Fuerzas Militares, se debe verificar este comportamiento al interior de otros sistemas de salud.

Es de resaltar para esta institución, que el Ejército y los pacientes de zona urbana de Cundinamarca aportaron más casos a esta casuística, especialmente durante los últimos meses del año, y en horario vespertino; períodos del día donde los pacientes se encuentran en su mayoría, al cuidado de sus padres o de su acompañante permanente, lo que está expresando la necesidad de un sistema de prevención y educación comunitaria.

De los resultados desprendidos de esta investigación, se propone la creación de un grupo que realice educación a padres y cuidadores, encaminada a la prevención de factores que modifiquen favorablemente el comportamiento del trauma.

Todo lo anterior servirá para dejar un precedente que permita a futuro contribuir con estos datos a un análisis multicéntrico a nivel nacional; y consolidar una base de datos del tamaño e importancia de las ya existentes a nivel nacional e internacional, que repercuta en la disminución de la incidencia y mortalidad de esta patología, la optimización de recursos humanos y de infraestructura. Esto es posible con la colaboración de todo el personal de salud, generando un completo desarrollo y registro de historias clínicas para sus posteriores análisis.

\section{Conflictos de interés}

Los autores declaran no tener conflicto de interés en la realización de este trabajo.

\section{Financiación}

Este trabajo se realizó con el apoyo financiero de los investigadores.

\section{Referencias}

1. Navascués del Ríos J.A, et al. Estudio Epidemiológico de los accidentes en la infancia: Primer Registro de Trauma Pediátrico. An Pediatric.1997;47(4).

2. Navascués J.A, et al. Registro de Trauma Pediátrico: Análisis de 1200 casos. Cir Pediatr.1998;11:151-160.

3. Correa M.A, González G,Herrera M.E, Orozco A
Epidemiología del trauma pediátrico en Medellín, Colombia 1992-1996. Colombia Med 2000;31 (2):77-80.

4. Indicadores básicos 2011.Situación de Salud en Colombia. Organización Panamericana de la Salud. Ministerio de Salud y Protección social República de Colombia. http://achc.org.co/documentos/investigacion/estadisticas/s ector/externas/OPS/10.IndicadoresBasicosdeSalud.Colomb ia2011

5. Comportamiento de muertes y lesiones por accidente de transporte, Colombia, 2013. Instituto Nacional de Medicina Legal y Ciencias Forenses. 2013. http:// www.medicinalegal.gov.co/documents/10180/188820/FO RENSIS+2013+4-+accidentes+de+transporte.pdf/ 51867e30-9ab5-4a15-8363-f2232d2c86ae

6. Tepas JJ, Mollitt DL, Talbert JL, et al . Pediatric Trauma Score as a predictor of injury severity in the injured child. J Pediatr Surg. 1987;22:14-18.

7. Tepas JJ, Ramenofsky ML, Barlow B , et al. National Pediatric Trauma Registry. J Pediatr Surg. 1989;24:156-8

8. Ramenofsky ML,Ramenofsky MB, Jurkovich GJ, et al. The predictive validity of the Pediatric Trauma Score. J Trauma 1988;28:1038-42

9. Tepas JJ, Ramenofsky ML, Mollitt DL, et al. The Pediatric Trauma Score as a predictor of injury severity: an objective assessment. J Trauma. 1988; 28:425-9.

10. Beaver BL, Haller JA. Epidemiología del traumatismo. En: Holder Thomas M. Ashcraft Keith W (eds.) Cirugía Pediátrica, Traducción de Pediatric Surgery. 2a ed. Philadelphia:Saunders Company; 1993.p108-115.

11. Iñón AE. Trauma en pediatría. Rev Cir Inf. 1994;4:5-7.

12. King DR. Trauma in infancy and childhood: initial evaluation and management. Pediatr Clin North Am 1985;32:1299-310.

13. American college of surgeons. Advanced Trauma Life Support (ATLS) student course manual. 7a ed. Chicago: American College of Surgeons; 2004

14. Alvarez Fernando, Figueroa LM. Trauma Pediátrico. Cinemática del Trauma. En: Fernando Alvarez López (ed).Libro Electrónico de Cirugía Pediátrica. Sociedad Colombiana de Cirugía Pediátrica. Facultad de Ciencias para la Salud. Departamento Clínico quirúrgico.Universidad de Caldas. s.f. http://www.sccp.org.co/plantillas/libroSCCP. 